\title{
Volume de iogurte light e sensações subjetivas do apetite de homens eutróficos e com excesso de peso
}

\section{(}

\section{Volume of light yogurt and subjective appetite}

sensations in normal-weight and overweight men

\author{
Luciana Neri NOBRE ${ }^{1}$ \\ Josefina BRESSAN² \\ Paulo de Souza COSTA SOBRINHO ${ }^{3}$ \\ Neuza Maria Brunoro COSTA2 \\ Valéria Paula Rodrigues MININ ${ }^{4}$ \\ Paulo Roberto CECON ${ }^{5}$
}

\section{RE S U M O}

\section{Objetivo}

Avaliar, independentemente de outras variáveis, o efeito do volume de iogurte light sobre os parâmetros de ingestão alimentar de homens saudáveis.

\section{Métodos}

Foi feita incorporação de ar ao iogurte por adição de um produto comercialmente disponível -Emustab ${ }^{\circledR}$ - $(6 \mathrm{~g} /$ $300 \mathrm{ml}$ ) com posterior homogeneização em liqüidificador semi-industrial. Utilizaram-se três volumes de iogurte: 300, 450 e 600ml. Trabalhou-se com 20 participantes saudáveis, sendo 10 eutróficos, com índice de massa corporal entre 19 e $24,9 \mathrm{~kg} / \mathrm{m}^{2}$ e 10 com excesso de peso, índice de massa corporal $\geq 25 \mathrm{~kg} / \mathrm{m}^{2}$. Cada um deles recebeu um volume de iogurte em três diferentes dias, pela manhã, em jejum de 12 horas. Uma escala de analogia visual foi utilizada, num período de 4 horas e 30 minutos após ingestão de cada volume do iogurte, para avaliar sensações subjetivas de saciedade, fome e desejo por alimentos específicos.

\section{Resultados}

Os volumes do iogurte light afetaram a saciedade dos dois grupos estudados, sendo que o maior volume exerceu melhor essa ação $(p<0,01)$. O maior escore para fome foi observado após ingestão do volume de

\footnotetext{
1 Departamento de Nutrição, Universidade Federal dos Vales do Jequitinhonha e Mucurí. Rua da Glória, 187, sala 34 Centro, 39100-000, Diamantina, MG, Brasil. Correspondência para/Correspondence to: L.N. NOBRE. E-mail: <lunerinobre@yahoo.com.br>.

2 Departamento de Nutrição e Saúde, Universidade Federal de Viçosa. Viçosa, MG, Brasil.

3 Faculdade de Ciências Farmacêuticas, Departamento de Alimentos e Nutrição Experimental, Universidade de São Paulo. São Paulo, SP, Brasil.

4 Departamento de Tecnologia de Alimentos, Universidade Federal de Viçosa. Viçosa, MG, Brasil

5 Departamento de Genética e Melhoramento, Universidade de Viçosa. Viçosa, MG, Brasil.
} 
$300 \mathrm{ml}$, seguido de 450 e $600 \mathrm{ml}(p<0,01)$. O desejo por alimentos doces, salgados, gordurosos e lanches não foi influenciado pelo tempo e volume do iogurte em ambos os grupos estudados. A ingestão energética subseqüente ao experimento não diferiu estatisticamente entre os dias do estudo e o dia sem iogurte $(p<0,05)$.

\section{Conclusão}

Os resultados deste estudo sugerem que o volume de iogurte light, independentemente de outras variáveis, pode afetar a fome e a saciedade.

Termos de indexação: fome; ingestão de alimentos; iogurte; obesidade.

\section{A B S T R A C T}

\section{Objective}

This study aimed to evaluate the effect of light yogurt volume on the food intake of healthy men regardless of other variables.

\section{Methods}

Air was added to the yogurt by mixing it with a commercially available product, Emustab ${ }^{\circledR},(6 \mathrm{~g} / 300 \mathrm{ml})$ and homogenized in a semi-industrial blender. Three volumes of yogurt were used: 300, 450 and 600ml. Twenty healthy volunteers participated in the study, 10 with normal weight, Body Mass Index from 19 to $24.9 \mathrm{~kg} / \mathrm{m}^{2}$ and 10 with excess weight, Body Mass Index $\geq 25 \mathrm{~kg} / \mathrm{m}^{2}$. Each one of them was given a yogurt volume in three different days in the morning, after a 12 hour fast. After the intake of each yogurt volume, a visual analog scale was used to assess the subjective sensations of satiety, hunger and desire for specific foods.

\section{Results}

Light yogurt volumes affected the satiety of both studied groups and the greater volume was more effective $(p<0.01)$. The highest hunger score was obtained after the $300 \mathrm{ml}$ intake, followed by 450 and $600 \mathrm{ml}(p<0.01)$. The desire for sweet, salty and fatty foods and snacks was not influenced by time and yogurt volume in either studied group. Energy intake between the days which yogurt was consumed and yogurt was not consumed was not statistically different $(p<0.05)$.

\section{Conclusion}

The results of this study suggest that the volume of light yogurt, regardless of other factors, may influence appetite and satiety.

Indexing terms: hunger; eating; yogurt; obesity.

\section{N T R O D U Ç Ã O}

Por ser uma doença em crescente ascensão, a natureza e as causas da obesidade são, atualmente, tópicos relevantes de pesquisa. Freqüentemente, o fenômeno ocorre como expressão da ingestão excessiva de calorias contidas nos alimentos, ingestão que supera o consumo energético, ocorrendo, portanto, quando se perde o equilíbrio entre a ingestão e o gasto de energia ${ }^{1,2}$. Porém, é difícil estabelecer se esse desequilíbrio resulta do excesso de ingestão ou de defeito no gasto energético ${ }^{1}$. Com relação à ingestão de alimentos, alguns dos fatores que propiciam o desencadeamento da obesidade podem ser o grande consumo de alimentos com elevada densidade energética ${ }^{2,3}$, a elevada ingestão de lipídeos ${ }^{3,4}$, assim como a baixa ingestão de fibras ${ }^{5,6}$.

Segundo Gatenby et al. ${ }^{7}$, Gatenby et al. ${ }^{8}$ e Arsenault \& Cline ${ }^{9}$, a partir da década de 80 , têm aumentado as estratégias para diminuir a incidência dessa patologia, e o consumo de produtos com baixo valor energético vem fazendo parte do cardápio diário de grande número de consumidores, tanto daqueles com excesso de peso quanto daqueles que almejam manter o peso corporal.

Embora os fatores supra citados, associados à obesidade, atuem simultaneamente, e ainda 
seja desconhecido o limiar de ação de cada um deles, um grande número de pesquisas vêm sendo desenvolvidas com o intuito de verificar os parâmetros de ingestão alimentar capazes de induzir maior saciação e saciedade. A saciação é o processo desenvolvido durante a alimentação e que culmina no término da ingestão $0^{3,6,10,11}$, enquanto que a saciedade inclui a diminuição da fome e a supressão da ingestão de alimentos na refeição seguinte ${ }^{3,6,10,12}$.

Segundo Douglas ${ }^{13}$, fome é o conjunto de sensações despertadas pela necessidade de alimento, que levam o indivíduo à procura, captação e ingestão do alimento, sendo a procura por alimento uma necessidade energética, biológica e não emocional. Para esse mesmo autor, o apetite é o desejo físico, ou emocional, de comer certo alimento específico. Trata-se de uma sensação dirigida a satisfazer um estado afetivo, que procura preencher mais uma necessidade emocional, ou seja, come-se porque existe o gosto pela comida, e isso produz satisfação emocional.

Entre os fatores relacionados com a ingestão, o volume do alimento consumido tem sido bastante estudado. Sabe-se que a distensão do estômago é um importante sinal de saciedade. Embora muitas pesquisas indiquem que a distensão gástrica possa parar o ato de comer ${ }^{14,15}$, segundo Blundel ${ }^{10}$ esse efeito tem curta duração.

Tendo em vista a importância da dieta, tanto no desenvolvimento como no controle da obesidade, e as pesquisas realizadas sobre o efeito do volume do alimento na ingestão alimentar, a proposta deste trabalho é estudar, isoladamente, a influência do volume de iogurte light sobre os parâmetros de ingestão alimentar em indivíduos eutróficos e com excesso de peso.

\section{MÉTODOS}

Este estudo foi desenvolvido com 20 participantes do sexo masculino com idade entre 18 e 40 anos, sendo 10 eutróficos, com índice de massa corporal (IMC) entre 19 a $24,9 \mathrm{~kg} / \mathrm{m}^{2}$, e
10 indivíduos com excesso de peso, IMC acima de $25 \mathrm{~kg} / \mathrm{m}^{2}$. O IMC foi avaliado segundo critérios da Organização Mundial da Saúde ${ }^{16}$.

O recrutamento foi feito por meio de cartazes em quadro de avisos nos departamentos da Universidade Federal de Viçosa (UFV) e por divulgação oral a estudantes e funcionários dessa instituição. Fez-se, por telefone, uma breve entrevista com o intuito de verificar se o possível candidato se encontrava apto para participar do experimento, pois esse deveria ser do sexo masculino, não ser fumante, não ter perdido ou ganhado mais de $3 \mathrm{~kg}$ nos últimos 6 meses; ter idade entre 18 e 40 anos e não estar fazendo dieta para controle de peso. A escolha do sexo masculino permitiu contornar as possíveis variações hormonais que, no sexo feminino, ocorrem com maior intensidade, o que poderia afetar a avaliação da ingestão alimentar.

Todos os potenciais participantes responderam a dois questionários. O primeiro questionário investigou a presença de algum tipo de alergia, aversão e preferência de sabores do iogurte a ser testado, assim como histórico clínico e presença de doenças na família: obesidade, hipertensão, diabetes e dislipidemia. O segundo ${ }^{17}$, Eating Inventary, teve o objetivo de avaliar restrição alimentar - possível escore: 0 a 21 -, desinibição - possível escore: 0 a 16 - e percepção da fome - possível escore: 0 a 14 - e não foi utilizado como fator eliminatório dos participantes, mas como uma forma de melhor conhecer seu comportamento alimentar dos mesmos. Os participantes que não atenderam aos critérios de inclusão foram excluídos da amostragem.

Os participantes foram informados que 0 objetivo do estudo era examinar o efeito do iogurte no apetite, porém não lhes foi dito que tipo de iogurte estava sendo ingerido e nem que a variação dos volumes se deu pela incorporação de ar. Todos assinaram um termo de consentimento para a realização do experimento, previamente aprovado pelo Comitê de Ética em Pesquisa com Seres Humanos da UFV. 
A avaliação antropométrica dos participantes do estudo foi realizada na Divisão de Saúde, UFV. Foram aferidas medidas de altura e peso para obtenção do IMC, classificado, posteriormente, de acordo com a Organização Mundial de Saúde ${ }^{16}$. O índice de massa corporal, que é a relação entre o peso do indivíduo em $\mathrm{kg}$ pelo quadrado da altura em metros, foi utilizado para classificação dos participantes como eutróficos e acima do peso.

Apesar de o IMC não ser considerado um bom indicador do estado nutricional, visto que não distingue massa gordurosa de massa magra, optou-se por classificar o estado nutricional dos participantes segundo esse indicador, por ser ele o mais utilizado para esse tipo de estudo. Para avaliação do peso utilizou-se uma balança eletrônica microdigital com capacidade de $150 \mathrm{~kg}$ e precisão de $100 \mathrm{~g}$, e para medida da altura, um antropômetro vertical milimetrado. A determinação do bom estado de saúde foi avaliada por meio de exames bioquímicos: hemograma completo em equipamento automatizado; colesterol total (BiolabMérieux, COD-011.104) e frações do colesterol HDL (BiolabMérieux, COD-111.531), LDL (BiolabMérieux, COD-111.532); triacilgliceróis (BiolabMérieux, COD-011.108); glicose de jejum (BiolabMérieux, COD-011.102); uréia (BiolabMérieux, COD-011.105); creatinina (BiolabMérieux, COD-011.107) e ácido úrico (BiolabMérieux, COD-011.109). Esses exames foram realizados no Laboratório de Análises Clínicas Santa Rita, na cidade de Viçosa, MG.

O experimento ocorreu na Cozinha Pedagógica do Departamento de Nutrição e Saúde da UFV. A incorporação de ar se deu pela adição de um emulsificante, Emustab ${ }^{\circledR},(6 \mathrm{~g} / 300 \mathrm{ml}$ de iogurte) ao iogurte light semicongelado e com posterior homogeneização em liquidificador semiindustrial. Os tempos utilizados para a incorporação de ar foram de 1, 3 e 5 minutos para os volumes de 300,450 e $600 \mathrm{ml}$, respectivamente. O Emustab ${ }^{\circledR}$ utilizado no experimento foi pesado em balança digital mini-slin, modelo $n^{\circ} 1475$, da marca Tanita ${ }^{\oplus}$. O processo de incorporação de ar variou o volume sem provocar alteração na densidade energética e no conteúdo calórico dos iogurtes. O iogurte light (IL) de marcas Danone ${ }^{\circledR}$, Brasil e o Emustab ${ }^{\circledR}$ Quatro Rodas ${ }^{\circledR}$, Brasil, utilizados no estudo, foram comprados em mercado local, sendo o iogurte light de sabor morango.

O experimento ocorreu em três dias não consecutivos, com intervalo médio, de uma semana, entre cada sessão de teste. Os volumes do iogurte foram oferecidos, aleatoriamente, a cada participante em cada dia de experimento. Os participantes apresentaram-se, no dia do teste, em jejum de 12 horas e foram orientados não só a fazer refeições nutricionalmente equilibradas durante os três dias que antecederam ao teste, como também a evitar esforço físico muito extenuante e uso de bebida alcoólica no dia anterior ao estudo.

Nesse tipo de estudo é difícil ter o controle total, por parte dos participantes, sobre o cumprimento das orientações fornecidas, uma vez que o voluntário não fica restrito a um laboratório durante todo o experimento. No entanto, para tentar assegurar o seguimento das orientações durante o processo de seleção, enfatizou-se a necessidade de segui-las em sua totalidade. Além disso, inquiriu-se cada participante, em todos os dias de estudo, sobre esse acompanhamento.

Os parâmetros de ingestão alimentar foram avaliados segundo metodologia adaptada de Flint et al. ${ }^{18}$, na qual se utiliza um bloco composto de sete perguntas com uma escala de analogia visual (EAV) de $10 \mathrm{~cm}$. As perguntas contidas no bloco foram: - Qual o grau de fome você sente agora? - Quão satisfeito você se sente agora? - Quão saciado você se sente agora? - Você gostaria de comer alguma coisa doce agora? - Você gostaria de comer alguma coisa salgada agora? - Você gostaria de comer algum salgadinho agora? - Você gostaria de comer alguma coisa gordurosa agora? Em cada extremidade da escala, houve uma opção de resposta que os participantes deveriam marcar conforme a sensação do momento em que foi oferecido cada bloco de questões. 
Para a avaliação dos parâmetros de ingestão alimentar, os participantes chegaram ao local do experimento por volta das 7h10min da manhã, nos três dias de estudo. Ainda em jejum, receberam informações sobre o uso da EAV e sobre o procedimento durante o teste e, em seguida, responderam ao primeiro questionário. As recomendações de como utilizar a EAV foram feitas em cada dia de teste. Após essa etapa, o iogurte foi servido em copo plástico leitoso descartável, sendo o volume de $300 \mathrm{ml}$ servido em copo de $300 \mathrm{ml}$; o de 450 , em copo de $500 \mathrm{ml}$ e o de $600 \mathrm{ml}$, em dois copos de $300 \mathrm{ml}$. A cada 30 minutos, novo questionário foi oferecido num período de 4h30min, totalizando 10 questionários para cada participante do estudo.

Durante o experimento, os participantes ficaram assentados em cadeiras ao redor de uma mesa. Entre eles não foi permitida discussão sobre as perguntas feitas durante a realização do teste, e nem conversa ou leitura de revistas sobre alimentos, a fim de evitar um estímulo à fome.

Para avaliação do seu consumo de energia, os participantes responderam a um recordatório alimentar de 24 horas e fizeram dois registros alimentares em dias aleatórios. Tal procedimento foi realizado a fim de obter uma média de três dias de ingestão de alimentos para melhor conhecimento da quantidade de energia ingerida pelos participantes antes do experimento. Em cada dia de estudo, eles também fizeram um registro dos alimentos sólidos e líquidos ingeridos, com sua respectiva quantidade em medidas caseiras. A partir desses valores, o valor médio de energia consumida antes do experimento foi comparado com os valores dos três dias do experimento. 0 cálculo da energia ingerida foi realizado utilizando-se o software DietPro ${ }^{\circledR}$ (versão 3.0).

Verificaram-se não apenas a correlação entre o IMC dos participantes e os parâmetros de ingestão alimentar, como também a presença de alguma interferência do volume do iogurte utilizado na saciedade e na ingestão alimentar nos dias do estudo.
Os dados foram analisados por meio de Análise de Variância (Anova) e Análise de Regressão, para verificar o comportamento dos grupos de participantes diante dos diferentes volumes de iogurtes sobre o desejo por alimentos específicos. Para verificar o impacto do volume de iogurte sobre a ingestão alimentar dos participantes, no almoço e nas 24 horas após o teste alimentar, utilizou-se o teste Dunnett para examinar diferenças entre as médias da ingestão nos três dias de experimento com o dia sem iogurte. Para os fatores quantitativos, os modelos foram escolhidos com base na significância dos coeficientes de regressão e no coeficiente de determinação do fenômeno em estudo. Os programas utilizados para as análises foram SAS for Windows - versão 6.12, SAS Institute Inc. Cary, N - e Sistema de Análises Estatísticas e Genéticas (SAEG) - versão 4.0.

\section{RESULTADOS E DISCUSS ÃO}

O perfil dos voluntários deste estudo constituiu-se da avaliação conjugada dos seguintes fatores: idade, altura, peso, IMC e o resultado do questionário Eating Inventory, com os valores médios e desvio-padrão (dp) (Tabela 1).

Os participantes apresentaram bom grau de instrução, uma vez que a maioria era consti-

Tabela 1. Características dos participantes, eutróficos e com excesso de peso, (média e desvio-padrão para $\mathrm{n}=10$ ). Viçosa, MG, 2002.

\begin{tabular}{lrrrrrr}
\hline \multirow{2}{*}{ Características } & \multicolumn{2}{c}{ Eutróficos } & & \multicolumn{2}{c}{ Acima do peso } \\
\cline { 2 - 3 } \cline { 6 - 7 } & \multicolumn{1}{c}{$\mathrm{M}$} & $\mathrm{DP}$ & & $\mathrm{M}$ & $\mathrm{DP}$ \\
\hline Idade (anos) & 25,40 & 5,00 & & 28,60 & 7,65 \\
Peso (kg) & 68,82 & 8,82 & & 103,68 & 21,20 \\
Altura (m) & 1,75 & 0,07 & & 1,76 & 0,09 \\
IMC (kg/m $\left.{ }^{2}\right)$ & 22,37 & 1,98 & & 33,00 & 5,03 \\
Eating Inventory & & & & & \\
Restrição Alimentar & & 8,70 & 3,95 & & 9,10 & 1,45 \\
Desinibição & 6,50 & 2,01 & & 8,30 & 2,36 \\
Percepção da fome & & 8,10 & 2,00 & & 5,90 & 3,00 \\
\hline
\end{tabular}

Notas: IMC=Índice de Massa Corporal; ${ }^{\mathbf{a}}=$ Mede intenção para dieta e pode medir casos de obesidade; ${ }^{\mathbf{b}}=$ Mede episódios de elevada ingestão alimentar; ${ }^{c}=$ Mede percepção da sensação de fome. 
tuída de estudantes da UFV, o que facilitou o estudo, já que o protocolo do experimento e algumas terminologias específicas da área de nutrição foram facilmente compreendidas. Esse fato também foi importante na confecção dos registros alimentares, pois é sabido que indivíduos com melhor grau de escolaridade lembram-se do alimento ingerido e o quantificam melhor.

O resultado do questionário Eating Inventary demonstra que os voluntários eutróficos e com excesso de peso apresentaram valores similares de restrição alimentar, sendo considerado de média restrição o escore de 6 a 9. Apresentaram, também, mesma classificação para desinibição - baixa desinibição: escore de 0 a 9 . Entretanto, o grupo eutrófico apresentou percepção da fome diferente, ou seja, apresentou melhor percepção da fome - escore de 5 a 7 que o grupo acima do peso. É importante destacar que, apesar de o grupo acima do peso ter apresentado a mesma classificação para desinibição que o grupo eutrófico, os integrantes deste grupo apresentaram menor valor - 6,5 $\mathrm{dp}=2,01$ - que aqueles do grupo acima do peso - 8,3 dp=2,36 -, indicando que, ao contrário dos eutróficos, o grupo acima do peso apresenta, possivelmente, freqüentes episódios de elevada ingestão alimentar.

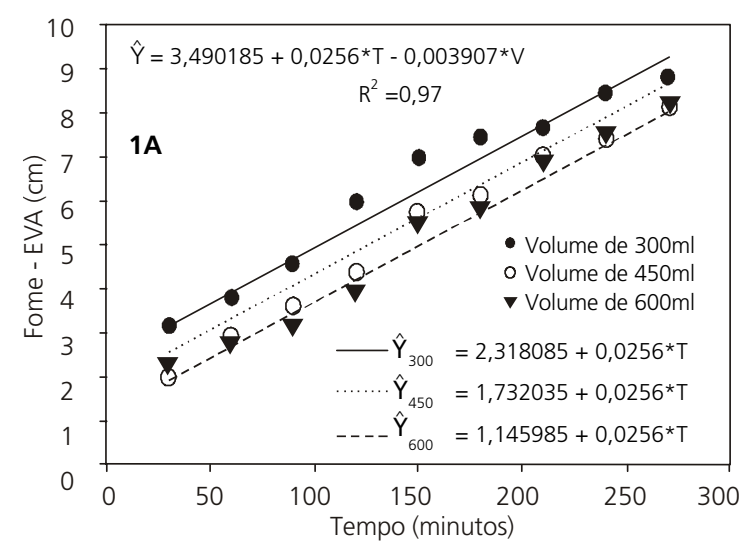

Quando foi avaliado o efeito do volume do iogurte sobre o desejo por alimentos específicos, tais como doces, salgados, gordurosos e salgadinhos, os resultados da estimativa do desejo dos participantes por esses alimentos mostraram que os volumes do iogurte light servido não interferiram, de forma substancial, no apetite para esses alimentos, ou seja, a estimativa do apetite para os alimentos citados não seguiu uma seqüência ordenada como, por exemplo, maior desejo após ingestão do menor volume de iogurte servido. Esse resultado era esperado, visto que a preferência alimentar é muito pessoal e não depende do volume do alimento ingerido.

Pode-se observar, pelas Figuras 1A e 1B, que a estimativa da fome foi crescente no decorrer do tempo - $p<0,01$-, no entanto, os participantes eutróficos relataram, inicialmente, pequena diferença na curva para essa sensação. Os eutróficos apresentaram valores absolutos similares após os diferentes volumes e valores maiores que os participantes acima do peso, e esses apresentaram curvas da sensação de fome diferentes para os três volumes do iogurte servido.

Esses dados confirmam o resultado do questionário Eating Inventory, aplicado neste estudo, o qual detectou que a percepção da

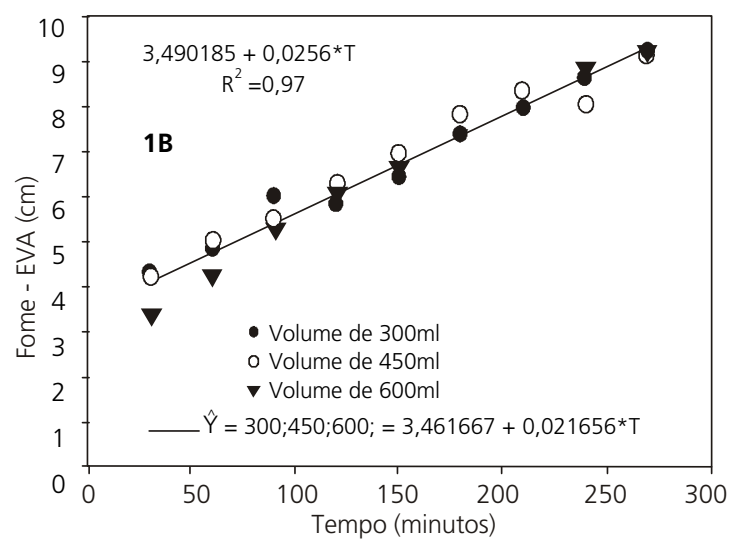

Figura 1. Estimativa da fome dos participantes acima do peso $(A)$ e eutróficos (B) após ingestão do iogurte light em função do tempo para os respectivos volumes. Viçosa MG, 2002.

Notas: * Significante em nível de $1 \%$ de probabilidade; $\hat{Y}=0$ valor da escala EAV para a fome, predita pela equação de regressão;

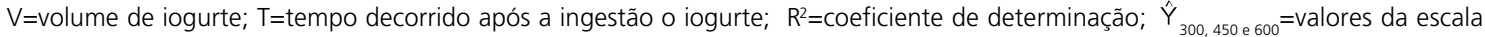
EAV para a fome preditos pela equação de regressão para o volume de iogurte de $300 \mathrm{ml}, 450 \mathrm{ml}$ e $600 \mathrm{ml}$, respectivamente. 
fome dos indivíduos eutróficos foi superior - 8,1 $\mathrm{dp}=2,0$ - à dos participantes acima do peso $(5,9$ $d p=3)$. Ao analisar esse dado, deve-se levar em consideração a reserva de tecido adiposo, que, segundo Blundell ${ }^{10}$, é um dos quatro domínios da atividade psicológica que contribuem para a expressão do apetite via sinal lipostático. Como o indivíduo magro tem, geralmente, uma reserva menor de massa adiposa, justifica-se por isso, maiores estimativas de fome nesse grupo.

Quando avaliada a estimativa da saciedade, essa foi decrescente no decorrer do tempo. No entanto, ao observar o comportamento dos participantes, os eutróficos apresentaram menores valores absolutos de estimativa da saciedade que os acima do peso. Embora não tenha sido verificada diferença estatística entre os volumes - $p<0,01$, houve, sim, uma tendência do maior volume desencadear melhor a saciedade, conforme demonstram as Figuras 2A e 2B.

Uma explicação provável para o volume do iogurte ter afetado a saciedade baseia-se na regulação da ingestão de alimentos, devido não só à estimulação mecânica exercida pelos nutrientes no estômago e no intestino ${ }^{19}$, e à ação de mecanorreceptores e quimiorreceptores no trato orofaríngeo e gastrointestinal ${ }^{20}$, como também à distensão e ao esvaziamento gástrico ${ }^{21}$.

Bell \& Rolls ${ }^{22}$ relatam que alimentos de grande volume induzem a uma percepção de que muita energia foi consumida; Pudel \& Oetting, citados por Porrini et al. ${ }^{21}$, relatam, ainda, que o comportamento alimentar das pessoas é influenciado tanto pelo que elas comem como pelo que elas acreditam que estão comendo. Para Bell \& Rolls ${ }^{22}$, as sugestões relacionadas à quantidade do alimento consumido têm, em curta duração, maior influência na ingestão que a quantidade de energia ingerida.

Avaliando-se a quantidade de energia ingerida pelos participantes do estudo durante $\mathrm{o}$ dia do experimento, observou-se que não houve diferença estatística - $p<0,05$ - entre o consumo de energia no almoço e nas 24 horas nos três dias do experimento, quando esse consumo foi comparado com o do dia sem iogurte-controle.

Os gráficos demonstrativos da ingestão de energia por grupo de participantes, durante os períodos de teste, podem ser vistos nas Figuras 3 e 4. Os participantes eutróficos apresentaram menor ingestão energética, quando o maior volume de iogurte - $600 \mathrm{ml}$ - foi servido. Esse grupo revelou, nas 24 horas, uma queda na ingestão de $11,36 \%$, quando essa ingestão foi comparada à do dia controle, mostrando, dessa forma, ter sido influenciada pelo volume do iogurte ingerido. Os participantes acima do peso, no entanto, apresentaram comportamento contrário, ou seja, o menor consumo de energia, 8,76\%, ocorreu após ingestão do menor volume de iogurte servido, $300 \mathrm{ml}$, apesar de esse grupo ter apresentado uma diminuição na ingestão energética em todos os dias de teste, quando comparada com a ingestão do dia controle.

O menor consumo de alimentos, pelos participantes eutróficos, após ingestão do maior volume do alimento teste, também foi observado por outros autores ${ }^{14,20,21}$ os quais acreditam que 0 volume, independentemente de outras variáveis, tais como densidade e estado físico, tem efeito sobre a saciedade e, conseqüentemente, sobre o consumo de energia e poderá ser utilizado como estratégia para controle de peso corporal.

É possível observar ainda, pelas Figuras 3 e 4, que, apesar de os valores absolutos do consumo de energia dos participantes eutróficos terem sido superiores aos dos participantes acima do peso, não foi encontrada diferença estatística do consumo entre ambos os grupos. Assim, parece que a composição corporal dos participantes do estudo não exerceu grande influência sobre a ingestão energética no almoço e nas 24 horas do dia do experimento. Esse resultado pode ser justificado pelo fato de os participantes terem ficado 12 horas de jejum antes do estudo, terem permanecido sem se alimentar por, aproximadamente, 5 horas após a ingestão dos iogurtes, e pelo valor calórico do iogurte servido ser muito baixo, $136,2 \mathrm{kcal}$, não sendo suficiente para manter um nível razoável de saciedade. 

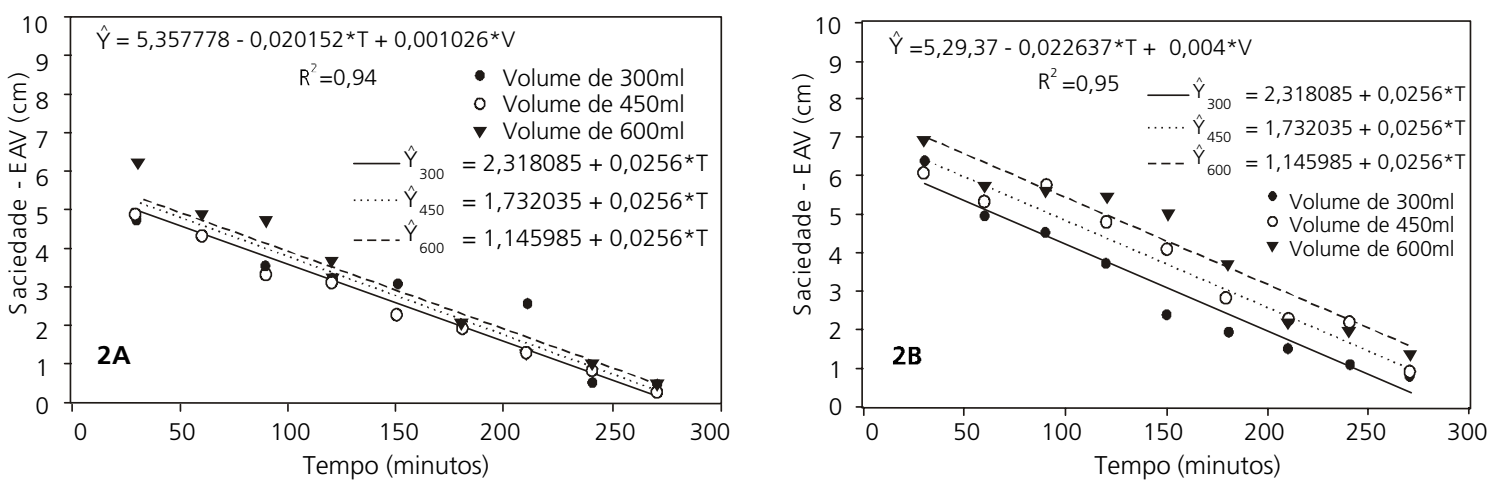

Figura 2. Estimativa da saciedade dos participantes acima do peso (a) e eutróficos (b) após ingestão do iogurte light em função do tempo para os respectivos volumes. Viçosa, MG, 2002.

Nota: *Significante em nível de $1 \%$ de probabilidade; $\hat{Y}=0$ valor da escala EAV para a saciedade predita pela equação de regressão;

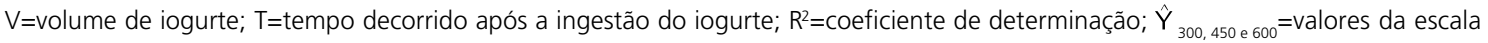
EAV para a saciedade preditos pela equação de regressão para o volume de iogurte de $300 \mathrm{ml}, 450 \mathrm{ml}$ e $600 \mathrm{ml}$, respectivamente.

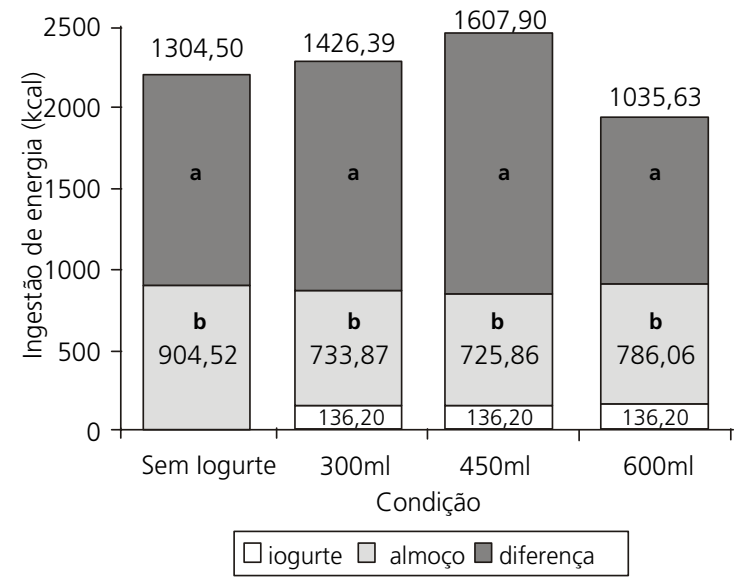

Figura 3. Média da ingestão de energia dos participantes eutróficos $(n=10)$ no almoço e nas 24 horas (diferença + almoço + iogurte) nos dias do teste em cada condição após ingestão do iogurte light. Viçosa, MG, 2002.

Nota: Médias com mesmas letras não foram estatisticamente diferentes em nível de $5 \%$ de probabilidade pelo teste Dunnett.

É importante ressaltar, também, que este experimento apresenta um protocolo diferente das pesquisas desenvolvidas nesta área. A grande maioria dos estudos sobre apetite utiliza pré-carga servida alguns minutos antes de uma refeição ${ }^{19,22-24}$, sendo, portanto, mais fácil verificar o impacto da pré-carga sobre a ingestão de

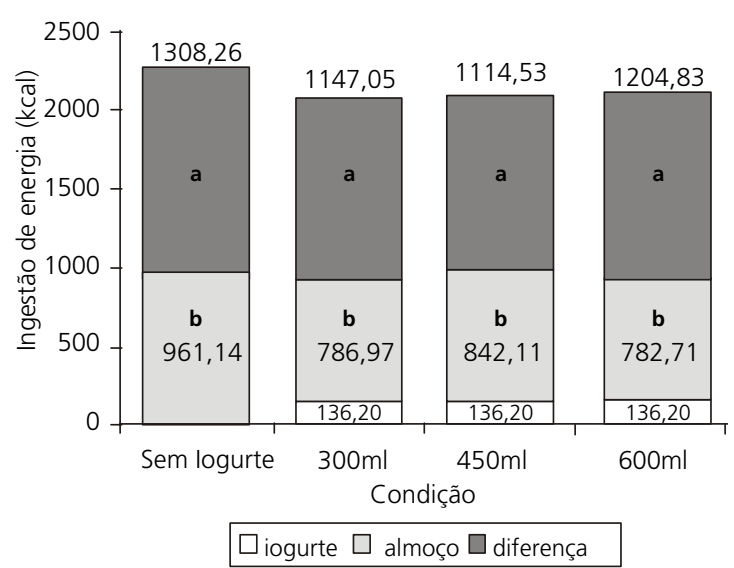

Figura 4. Média da ingestão de energia dos participantes acima do peso $(n=10)$ no almoço e nas 24 horas (diferença + almoço + iogurte) nos dias do teste em cada condição após ingestão do iogurte light. Viçosa, MG, 2002.

Nota: Médias com mesmas letras não foram estatisticamente diferentes em nível de $5 \%$ de probabilidade pelo teste Dunnett.

alimentos. Neste experimento, o iogurte servido não funcionou como uma pré-carga, mas como um desjejum de muito baixo valor energético.

Embora estudos anteriores tenham relatado que homens jovens e magros apresentem melhor compensação energética que homens obesos $^{25}$, isso não foi verificado neste experimento. 
Mesmo tendo os participantes apresentado IMC entre 19,29 a $39,95 \mathrm{~kg} / \mathrm{m}^{2}$ e idade entre 18 a 40 anos, observou-se que os grupos acima do peso e eutróficos se comportaram similarmente, ou melhor, a composição corporal e a idade não tiveram influência estatisticamente significante sobre a ingestão de alimentos no almoço e nas 24 horas no dia do experimento.

Segundo Serra-Majem \& Ribas-Barra ${ }^{26}$, a habilidade para fazer um registro alimentar pode ser influenciada pela idade, pelo gênero e pelo nível de escolaridade, e os homens, ao contrário das mulheres, são menos atenciosos quanto à quantificação de alimentos ingeridos. Assim, apesar de ser questionada a confiabilidade do registro alimentar, ele é um dos recursos bastante utilizados para a realização desse tipo de estudo fora de um ambiente de laboratório. E neste experimento, para ter uma maior confiabilidade do registro, cada um foi revisado individualmente com os participantes, para verificar se as informações estavam completas e se algum item não esclarecido foi identificado.

\section{CONCLUSÃ O}

Os resultados deste estudo mostram que, nas condições deste experimento, o volume do iogurte light, parece afetar a saciedade e os parâmetros de ingestão alimentar, independentemente da densidade calórica. No entanto, em todas as condições deste estudo, não se observou diferença significativa nas sensações subjetivas de fome, saciedade e desejo por alimentos específicos. O peso corporal não exerceu influência significativa na preferência por alimentos específicos e na ingestão calórica no almoço e nas 24 horas do dia do experimento. Assim, os resultados deste estudo sugerem que o consumo de alimentos de grande volume pode ser efetivo no desencadeamento da saciedade e, talvez, na indução de um menor consumo de energia, especialmente para pessoas eutróficas. Estudos de longo prazo sobre o efeito do volume do alimento, independentemente de outras variáveis, como estado físico e densidade calórica, são, entretanto, necessários para que se possa esclarecer quanto às suas indicações na prática clínica.

\section{REFER Ê N CIAS}

1. Monteiro CA, Halpern A. Epidemiología de la obesidad en Brasil. Nutr Obes. 2000; 3(2):98-105.

2. McCrory AM, Fuss PJ, Saltzman E, Roberts SB. Dietary determinants of energy and weight regulation in healthy adults. J Nutr. 2000; 30(2): 276S-9S.

3. Rolls BJ. The role of energy density in the overconsumption of fat. J Nutr. 2000; 130(2): 268S-71S.

4. Hill JO, Melanson EL, Wyatt H. Dietary fat intake and regulation of energy balance implication for obesity. J Nutr. 2000; 130(2):284S-8S.

5. Duarte HS, Monteiro JBR, Costa NMB. Efeito de uma sopa rica em fibra sobre a ingestão alimentar, peso e composição corporal. Rev Bras Nutr Clin. 1999; 14:228-38.

6. Burton-Freeman B. Dietary fiber and energy regulation. J Nutr. 2000; 30(2):272S-5S.

7. Gatenby SJ, Aaron JI, Morton GM, Mela DJL. Nutritional implications of reduced-fat food use by free-living consumers. Appetite. 1995; 25(3): 241-52.

8. Gatenby SJ, Aaron JI, Jack VA, Mela DJL. Extended use of foods modified in fat and sugar content: nutritional implications in a free-living female population. Am J Clin Nutr. 1997; 65(6):867-73.

9. Arsenault LE, Cline AD. Nutrient intakes characteristics of normal weight, female military personnel consuming foods reduced in fat our energy content. Appetite. 2000; 34(3):227-33.

10. Blundell J. A fisiologia do controle do apetite. In: Halpern A, Matos AG, Suplicy HL, Mancini MC, Zanella MT. Obesidade. São Paulo: Lemos Editorial; 1998. p.103-11

11. Burns AA, Livingstone MBE, Welch RW, Dunne A, Robson PJ, Lindmark L, et al. Short-term effects of yoghurt containing a novel fat emulsion on energy and macronutrient intake in non-obese subjects. Int J Obes. 2000; 24(11):1419-25.

12. Blundel J, Green SM. Effect of sucrose and sweeteners on appetite and energy intake. Int J Obes. 1996; 20(Suppl.2):S12-S17. 
13. Douglas CR. Controle da ingestão alimentar. In: Douglas CR. Tratado de fisiologia aplicada à nutrição. São Paulo: Robe; 2002. 1046p.

14. Phillips RJ, Powley TL. Gastric volume rather than nutrient content inhibits food intake. Am J Physiol Regul Integr Comp Physiol. 1996; 271(3): R766-R79.

15. Jones KL, Doran SM, Hveem K, Bartholomeusz FD, Morley JE, Sun WM, et al. Relation between posprandial satiation and antral area in normal subjects. Am J Clin Nutr. 1997; 66(1):127-32.

16. World Health Organization. Obesity: Prevention and managing the global epidemic. Repot of a WHO Consultation on Obesity. Geneva; 1998.

17. Stunkard AJ, Messick S. The three-factor eating questionnaire to measure dietary restraint, disinhibition and hunger. J Psychosom. 1985; 29(1):71-83.

18. Flint RA, Blundell J, Astrup A. Reproducibility, power and validity of visual analogue scales in assessment of appetite sensations in single meal studies. Int J Obes. 2000; 24(1):38-48.

19. Rolls BJ, Castellanos VH, Halford JC, Kilara A, Panyam D, Pelkman $C L$, et al. Volume of food consumed affects satiety in men. Am J Clin Nutr. 1998; 67(6):1170-7.

20. Geliebter A. Gastric distension and gastric capacity in relation to food intake in humans. Physiol Behav. 1988; 44(4-5):665-8.
21. Porrini M, Santangelo $A$, Crovetti R, Riso $P$, Testolin $G$, Blundell JE. Weight, protein, fat and timing of preloads affect food intake. Physiol Behav. 1997; 62(3):563-70.

22. Bell EA, Rolls BJ. Energy density of foods affects energy intake across multiple levels of fat content in lean and obese women. Am J Clin Nutr. 2001; 73(6):1010-8.

23. Graaf $C$, Hulshof T. Effects of weight and energy content of preloads on subsequent appetite and food intake. Appetite. 1996; 26(2):139-51.

24. Rolls BJ, Bell EA, Waugh B. Increasing the volume of a food by incorporating air affects satiety in men. Am J Clin Nutr. 2000; 72(2):361-8.

25. Rolls BJ, Kim-Harris S, Fischman MW, Foltin RW, Moran TH, Stoner SA. Satiety after preloads with different amounts of fat and carbohydrate: implications for obesity. Am J Clin Nutr. 1994; 60(4):476-87.

26. Serra-Majem L, Ribas-Barra L. Recordatório 24 horas. In: Serra-Majem L, Aranceta-Bartrina J, Mataix-Verdú J. Nutrición y Salud Pública Métodos, bases científicas y aplicaciones. Barcelona: Masson; 1995. p.113-9.

Recebido em: 8/3/2004

Versão final reapresentada em: 4/7/2006 Aprovado em: 4/8/2006 